\title{
REFERENTIAL USES OF ARABIC NUMERALS
}

\author{
Melissa Vivanco \\ https://orcid.org/0000-0001-9923-7208 \\ University of Miami \\ Department of Philosophy Miami, FL \\ U.S.A. \\ melisa.viva@ciencias.unam.mx
}

\begin{abstract}
Article info
CDD: 401

Received: 25.08.2020; Revised: 09.09.2020; Accepted: 14.09.2020

https://doi.org/10.1590/0100-6045.2020.V43N4.ME
\end{abstract}

\section{Keywords}

Arabic numerals

Reference

Arithmetical epistemology

Numbers

Abstract: Is the debate over the existence of numbers unsolvable? Mario Gómez-Torrente presents a novel proposal to unclog the old discussion between the realist and the anti-realist about numbers. In this paper, the strategy is outlined, highlighting its results and showing how they determine the desiderata for a satisfactory theory of the reference of Arabic numerals, which should lead to a satisfactory explanation about numbers. It is argued here that the theory almost achieves its goals, yet it does not capture the relevant association between how a number can be split up and the morphological property of Arabic numerals to be positional. This property seems to play a substantial role in providing a complete theory of Arabic numerals and numbers. 


\section{THE GAME: HOW NUMERALS COULD REFER INSTEAD OF WHETHER NUMERALS REFER}

There is a well-known debate about the metaphysics of natural numbers. Typically, the discussion takes place in a match whose players belong to one of two predefined teams: the Realistand the Antirealist. If you choose the Realist team, as Frege (1884), Burgess and Rosen (1997), Hale and Wright (2009), and others have done, prepare yourself to commit to the existence of natural numbers as abstract, objective, and (not necessarilybutmostlikely) mind-independententities. The realist player holds that arithmetical sentences are true in virtue of facts about the denotations of their singular terms and predicates. Her challenge in this game is to explain by virtue of what do we gain knowledge of arithmetic sentences (since we don't have the same type of contact with abstract entities as we do with whatever entities that are supposed to make empirical sentences true). Naturally, you might like the Antirealist team better. The spirit of this popular team is to deny the existence of entities such as numbers (see Field (1989), Yablo (2010), Bueno (2016) Once you choose to become an antirealist, your challenge is to explain in virtue of what are arithmetical sentences true. This game has spawned a diverse variety of accounts in which each team shows off their most sophisticated tactics, even reaching extreme positions with consequences such as that the only possible result is that both teams 'win' (for example, defending that only radical realism and radical antirealism are tenable, as Balaguer (1998) does) or that both teams 'lose' (as in a case of unsolvable epistemic disagreement (see Rosen (2001)).

In the fourth chapter of Roads to reference, Gómez-Torrente (2019) presents an attractive and novel account where the starting point is to put aside the traditional game- which has come to seem bogged down-and starts a new one. The opening move of this game consists of taking at face value our linguistic intuitions bearing on the question of how the 
referents of the numerals could get fixed.

The interesting question in this contextis not, I think, whether skeptical or nominalist doubts can be assuaged to the full satisfaction of the doubters, but rather whether there is an intuitive ontology and epistemology of the numbers and the numerals that can underwrite a sensible account of reference fixing for the numerals. (p.108)

The winner of the new game will be the one who can provide an explanation of the reference fixing of the numerals that can be adequately accompanied by a plausible ontology and a satisfactory epistemology about numbers. This is the objective that Gómez-Torrente tries to achieve in this chapter.

\section{THE TEAMS: DESCRIPTIVISTS VS. R EFERENTIALISTS}

As Gómez-Torrente points out, there are two kinds of accounts of Arabic numerals that are seriously considered in the literature on reference. These are the Descriptivist team, which holds that Arabic numerals have semantic structure, being semantically equivalent with certain mathematical descriptions or related phrases, and the Referentialist team, which holds that Arabic numerals are not equivalent with descriptions and are instead semantically unstructured singular terms such as proper names, pure indexicals and simple demonstratives are likely to be.

Along with other non-descriptiveness semantic views developed in the book, Gómez-Torrente argues that numbers are not descriptions. From the referential side on numerals, the first move consists of ruling out the most prominent 
descriptivist semantics. The strategy is to analyze paradigmatic examples of different descriptivisms and raise objections that would presumably span classes of the most plausible theories of this kind.

As said, the procedure to fix the reference of Arabic numerals is not completely description-free. The author is pushed to adopt a kind of descriptivist 'fixes- the-reference' view. Nonetheless, since the reference of an Arabic numeral is not a description itself, this procedure must be carefully carried out.

\subsection{Fixing the reference by verbal numerals}

The defender of this view holds that Arabic numerals either abbreviate or have their reference conventionally fixed by their corresponding verbal numerals. If so, the advocate of semantic non-descriptivism for Arabic numerals is about to lose because once she accepts a method of fixing the reference using complex verbal numerals, there seem to be no better candidates for the semantic values of Arabic numerals than those or very similar descriptions. In such a view, '765' abbreviates or has its reference conventionally fixed by a description such as '(seven (times) (one) hundred) (plus) (sixty (plus) five)'. What Gómez-Torrente argues is that there are many more intelligible Arabic numerals than the conventional verbal numerals in anytypical speaker's idiolect: eventually, we would run out of verbal numerals to fix the references of Arabic numerals.

Another problem with this crude version of the view is that it might lead to certain classic epistemological puzzles. Imagine that a friend of mine from France, Margot, and I are competent users of Arabic numerals but are not very well versed in arithmetic. Margot knows that '99' stands for quatre-vingt-dixneuf whose corresponding description in English would be 
something like (four (times) twenty (plus) ten (plus) nine). On the other hand, I know that '99' stands for (ninety (plus) nine). The statement ' 99 is (ninety (plus) nine)' is necessarily true and I know it a prior; Margot knows a priori that ' 99 is (four (times) twenty (plus) ten (plus) nine)' is true, this identity is also necessary; we both know that ' $99=99$ ' is true, but we may not know that '(ninety (plus) nine) is (four (times) twenty (plus) ten (plus) nine)' is true. Would we say in this case that Margot and I know the semantic value of ' 99 '?

The advocate of fixing the reference by verbal numerals has to explain how the finite resources from verbal numerals can be sufficient to fix the references of the infinite Arabic numerals, as well as the relation between the descriptions used to fix the references and the semantic values of the Arabic numerals, in such a way that epistemic problems such as those mentioned above are avoided. The ball is in their court.

\subsection{Two more moves by the Descriptivist}

What is relevant for current purposes is that GómezTorrente builds his own referential strategy based on rules deployed from descriptivist moves. Due to space limitations, I present broadly the two most prominent descriptivisms ${ }^{1}$ that Gómez Torrente addresses and highlight the rules that are derived from them.

$$
\begin{aligned}
& D_{P 2} \text { Arabic numerals are semantically } \\
& \text { equivalent with certain } \\
& \text { mathematical descriptions, }
\end{aligned}
$$

1 Gómez-Torrente mentions other theories, as well as other variations of these ones, and he provides arguments to show that all of them are challenged by the same sort of objections. 
generally polynomial expansions

such as ${ }^{\prime} 5(9+1)^{0}+$

$6(9+1)^{0+1}+7(9+1)^{0+1+1}$,

in the case of ' 765 '.

$D_{P} 3$ The description giving the

meaning or fixing the reference of a numeral $N$ later than ' 0 ' in the natural order would be of the form of 'The number of numerals between ' 0 ' and ' $M$.' (Where for ' $M$ 'one would substitute the Arabic numeral coming just before $N$ in the natural order.)

The first move above exhibits the Kripkean antidescriptivism argument about the semantic detachment for names applied to numerals. This is analogous to the case where the name 'Gödel' still would refer to Gödel even if the description 'The discoverer of the incompleteness of arithmetic' were not satisfied by Gödel.

Suppose that Arabic numerals are semantically equivalent to polynomials such as the one used in $D_{P 2}$. In the case of natural numbers, exponentiation does not constitute a fundamental arithmetic operatio ${ }^{2}$, but rather an extension of multiplication

\footnotetext{
${ }^{2}$ Some mathematicians struggle with the fact that any number raised to the zero power is 1 . Some justifications can be provided: we can say that the zero power is just the product of no numbers at all, which is the multiplicative identity, or that pn is the number of functions from a set of cardinality $n$ to a set of cardinality $p$, if $\mathrm{n}=0$ the number of functions at play is 1 (the empty set); GómezTorrente offers another justification in the footnote 17. All these reasons carry a sense of artificiality to be mathematical grounds of the fact that $\mathrm{p} 0=1$ for all integers $\mathrm{p}$. Basically, what is said is that
} 
(even multiplication could be questioned since it is an extension of addition that is dispensable in the construction of the inductive structure of natural numbers). Given this consideration, we can imagine a case where someone discovered that the exponentiation of natural numbers is not well defined after all. Would this imply that a typical user of the Arabic numerals would have never referred to 765 by ' 765 ', given that the description $5(9+1)^{0}+6(9+1)^{0+1}+7(9+$ $1)^{0+1+1}$ ' would have turned out to determine no number? The answer seems to be that this would not be implied and ' 765 ' would still refer to 765 .

Two more observations from $D_{P 2}$ are noteworthy. Typical English speakers seem to have de reattitudes involving the natural numbers when they entertain certain attitudes via Arabic numeral representations (at least when the numerals are not too long), but not when they entertain them via other representations which are more clearly descriptive (see Ackerman (1978) and Kripke (1992)). This is the case of the polynomial in $D_{P 2}$.

One way to make our de re attitudes more apparent is by analyzing How-many questions. Standard theories of the semantics of interrogatives characterize questions as sets of answers, where an answer to a question is the semantic value of an expression that would count as a (correct or felicitous) response to the corresponding interrogative, (cfr. Groenendijk and Stokhof (1997); Krifka (2011)).

Imagine that I text Margot with the question 'How many customers went to your restaurant today?'. Con- sider the answers: a) ' 795 ', and b) ' $5(9+1)^{0}+6(9+1)^{0+1}+7(9+1)^{0+1+1}$ '. They both are correct in some sense. Nevertheless, the only one that is clearly felicitous is a), since this is the only one that

p0 $=1$ because otherwise un-desirable practical consequences would hold. So we must accept the fact as a necessary stipulation. 
rules out an invitation to another How-many question (which would reflect that the expectations of the asker have not been satisfied). If Margot texts me back b), I may very well have to ask her 'And how many are those?'

A felicitous answer to a How-many question (note from the example that the How-many question can be replaced by a What-is-the number-of question) seems to be the one that concerns our de re attitude toward the number that turns out to be the semantic value of the correctanswer. The best candidate is the answer with Arabic numerals. Now let's move on to the second observation.

According to Gómez-Torrente, what makes the polynomialin $D_{P} 2$ attractive with respect to others (for example, the polynomial whose existence is guaranteed by the Fundamental Theorem of Arithmetic) is that once the reference of the digits is fixed (and the meaning of the operations is established) the reference of complex Arabic numerals is determined. That seems to be a good reason to choose that kind of polynomial, but the way I see it, there is a deeper (related) reason to make that choice. The form of such a polynomial is reflected in the morphological nature of the corresponding numeral: the term whose power has one numeral determines the digit that occupies the first place fromright toleftinthenumeral, theterm whose power has two numerals occupies the second place from right to left in the numeral, and so on. For reasons explained in a subsequent section, I would say that this is a potential point in favor of $D_{P_{2}}$ and against the Gómez-Torrente's account. Indeed, the author acknowledges that complex Arabic numerals have a formal complexity that must somehow be relevant to their content (p.112).

$D_{P 3}$ move raises another concern. Some descriptivisms seem to be tooconceptually demanding to explain everyday uses of Arabic numerals (think of Margot and I having trouble with number communication). For example, people can be competent with numerals for specific numbers without having 
much arithmetical background or the general concept of a numeral, which is necessary to understand descriptions such as $D_{P 3}$. As Gómez-Torrentepoints out, these descriptions do not seem to be part of the semantic structure behind the numerals or even of the conceptual wherewithal of the users of the numerals as reference fixers.

To conclude, it is convenient to draw a final moral from descriptivism. According to the Kripkean anti-descriptivism arguments for standard proper names, the existence of a cognitive contact between the speaker and the referent makes the question of whether a certain object is that referent in the speaker's idiolect detachable from the question of whether that object satisfies those descriptions (this explains, for example, why in cases of faulty uses of descriptions the speaker still manages to refer). However, this can't generally be applied to cases in which a speaker-baptizer fixes the referent with the help of a description. Such a speaker will associate descriptive material with the name in an undetachable way. Unlike the paradigmatic Kripkean story about proper names, the procedure by which Arabic numerals obtain their reference does not appear to be completely free of descriptive content. If the Referentialist accepts a descriptivist 'fixes-the-reference' view, in order to guarantee a successful reference to numbers through the use of Arabic numerals, she must account for a cognitive contact between the speaker and the numbers that turns out to be at least in some degree detachable from descriptive content.

\section{THE RULES: NEW CHALLENGES FOR THE REFERENTIALIST}

As a response to descriptivist strategies, Gómez-Torrente presents a 'fixes-the-reference' descriptivist view of content fixing for the Arabic numerals that will appeal to less sophisticated 
descriptions, a view that will be accompanied by the postulation of a significant role for certain elements that can plausibly be said to provide a non-descriptive cognitive contact between speakers and numbers (p.115).

$R_{P 1}$ The correct view of the Arabic numerals is a descriptivist 'fixes-the-reference' view, but the numerals are not semantically equivalent with descriptions

The Referentialist move, $R_{P 1}$, must comply with the rules that have been set by the lessons learned from the descriptivist moves:

1. The detachment argument $\left(R_{P 1}\right.$ is exempt from this because the procedure of fixing the reference involves descriptive content)

2. The descriptions involved in the fixing-thereference procedure should not be sophisticated to a degree that requires extensive conceptual (linguistic or mathematical) resources on the part of the speaker

3. Complex Arabic numerals must get their interpretations by means of a general procedure which exploits in some way their morphological constituents and the meanings

4. The speaker must be successful in referring to the numbers in a way that is independent of the fact that the numbers satisfy the descriptions involved in the procedure for fixing the reference of Arabic numerals

5. The fixing-the-reference procedure must lead to an ontology and epistemology that accounts for our de re attitudes toward 
numbers through the use of Arabic numerals

\subsection{Fixing the reference}

As mentionedin $₫ 1$, the Realistused to be required to explain our epistemic access to what the semantic values of the numerals could be, which would ultimately explain our knowledge of arithmetic truths. Such a requirement cannot be overlooked by the Referentialist. In the new game, the procedure for fixing the reference of the Arabic numerals should at least partially help to provide a satisfactory answer to the question.

Gómez-Torrente's proposal is based on the existence of the ability to generate the series of Arabic numerals without recourse to sophisticated arithmetical knowledge:

A. Small Arabic numerals, like '1', get their referents either via translation to their corresponding verbal numerals, or directly via descriptions similar to those that presumably fix the referents of small verbal numerals, such as 'the number of these fingers', or 'this number'

B. Larger Arabic numerals get their referents fixed in a typical speaker's idiolect when she masters systematic ways to identify those referents in terms of the referents of the smaller numerals. For example, by means of the speaker's disposition to associate Arabic numerals (coming after ' 1 ' and following ' $M$ '), with descriptions of the form 'The number greater by one than $M^{\prime}$ 
Number Rule. The Arabic numeral ' 1 ' refers to the number one; and whenever an Arabic numeral refers to a certain number, the Arabic numeral that follows it in the generatingorder refers to the number greater by one than that number.

A competent user could in principle associate with each Arabic numeral with which she is acquainted with one or more particular utterances of reference-fixing descriptions for that numeral which intuitively follows from the Number rule. The ability to generate and interpret bigger numbers via the mechanism is, not coincidentally, related to the ability to count; it is ultimately this ability that provides our most basic conceptions of number (pp.127, 128).

This move to fix the reference of Arabic numerals suggests an answerto the epistemological question: access to numbers will be partially explained by certain abilities typically identified as linguistic, which can be combined with abilities of a different cognitive nature (for example, the ability to identify small multiplicities), and playing the ability to count-which plays a privileged role.

The mastery of these abilities eventually induces the appearance of particular conceptions of the numbers in a progressively larger set, and also of a minimal conception of the general notion of number that, according to Gómez-Torrente, includes the idea that the number of a multiplicity of things is an aspect of it that is common to other multiplicities of things that can be counted by means of the same verbal numeral (among others related ideas). Additionally, I would include the idea that a multiplicity of things can be arranged into smaller multiplicities of things, each of those corresponding to a verbal numeral; the relation between the smaller multiplicities and the total is associated with the relation between the verbal numerals that correspond to the smaller 
multiplicities and the verbal numeral that corresponds to the total. For instance, a multiplicity of 125 things whose corresponding numeral is 'one hundred twenty-five' can be arranged (in particular) into three multiplicities of things: one of 100 thingswhose corresponding numeral is 'one hundred', one of 20 things whose corresponding numeral is 'twenty', and one of 5 things whose corresponding numeral is 'five'.

\subsection{What the numbers could be}

For the ontological move, Gómez-Torrente offers a wide variety of reasons to support the claim that numbers are cardinality properties of pluralities. Contrary to other potential candidates of what numbers could be, these properties stand in the same relation as numbers do to the corresponding pluralities. I consider that this is a compelling reason to hold that numbers are cardinality properties of pluralities.

Provided the numbers are any things atall, a natural number must by its nature be susceptible of being had by pluralities of things, susceptible of being the number of pluralities. Thus, 17 is so susceptible, and in factitis the number of themajor moons of Jupiter. (Gómez-Torrente 2015, p. 317)

Whatever numbers are, there is an intrinsic relation between them and the pluralities that have them as their numbers. This relation is the same as that between cardinality properties (and only them) and any plurality that has them as properties. 


\subsection{Following the rules of the game?}

It is not difficult to concede that Gómez-Torrente's account does not face a major problem regarding rules 1,2 , and 4. In my view, rules 3 and 5 deserve more attention.

The question regarding rule 3 is, to what extent does the proposed fixing-the-reference procedure exploit the morphological components and meanings of the Arabic numerals?

To answer this question, it is worth recalling the basics of the procedure. Let's call this set $B$ :

- The speaker's general conception of number and of adding one

- The ability to generate and interpret bigger numbers via the NumberRule (related to the ability to count)

- Having the reference of ' 1 '

- Once the reference of ' $M$ 'has been fixed, the reference of the following numeral in the natural order ' $N$ ' is fixed in virtue of the fact that $N$ is greater by one than $M$

The procedure certainly reflects some relevant association between the morphological nature of numerals within a positional system of Arabic numerals and the numbers that constitute the structure of natural numbers. Namely, that the transition from ' $M$ ' to ' $N$ ' is attached to the transition from $M$ to $N$ given by adding 1 to $M$. The association captured by $B$ is reflected in the similarity between the procedure and what it takes and the characterization of natural numbers as the 
inductive set ${ }^{3}$ such that 1 is the first element, and the others are produced by adding 1 successively. Butwhatdoes thishaveto do with the morphological components and meanings of Arabic numerals? There seems to be no reason to think that the procedure would not work for other numbering systems that are essentially different from Arabic numerals. In particular, for systems in which numerals have a substantially different morphology. If so, it is very unclear that rule 3 is being followed. Furthermore, since the last rule requires that the theory at play accounts for our de re attitudes toward numbers via Arabic numerals, this also jeopardizes the move with respect to rule 5 .

The procedure may seem adequate for consecutive numerals whose morphology does not change significantly after the transition by adding 1 of the corresponding numbers. Consider for example, '764' and ' 765 ' (since ' 5 ' is the numeral that follows ' 4 ' in the natural order, whose referent is obtained from the fact that 5 is the number greater by one than 4), we have a straightforward account for the reference of ' $765^{\prime}$ in terms of their constituents. Nonetheless, the account seems to lack an explanatory element for cases like '999' and '1000' whose morphologies (determined by the constituents) differ more substantially. It seems that Gómez-Torrente's account underestimates the fact that Arabic numeral systems are positional, which is a salient aspect regarding their morphology. I think that unsurprisingly, this property is not only associated with a relevant characteristic of numbers but also plays an important role in how Arabic numerals manage to refer to numbers.

\footnotetext{
${ }^{3}$ A nonempty partially ordered set in which every element has a successor.
} 
To illustrate the aforementioned point, consider a nonpositional system (one in which the position a symbol occupies in the numeral bears no relation to its value ${ }^{4}$ ). For instance, the system where ' 1 ' stands for the number one, '11' stands for the number two, '111' stands for the number three, and so on. The procedure offered by Gómez-Torrente succeeds in accounting for how the numerals in this system get their references fixed. The speaker picks out the referent of ' 1111111111 ' in virtue of the fact that the numeral ' 1 ' refers to the number one and the ability to recognize that ' 1111111111 ' follows to ' 111111111 ' because its referent (the number ten) is the number greater by one than the referent of '111111111', which has been already fixed by the same procedure. That which constitutes $B$ is apparently all that is needed to explain how almost any system whose numerals refer to numbers manages to do so.

Certainly, such numerals do not seem to entertain de re attitudes toward the number 102 as thenumeral '102' does in the positional decimal Arabic system. (Or as the numeral '1212' allegedly would do if we had been trained in the Arabic numeral system in base 4). If I ask Margot how many costumers went to her restaurant today and she texts me a sequence of 765 ones, this does not seem to be a felicitous answer to my question: either I have to count the quantity of ones or text her again 'And bow many are those?'.

${ }^{4}$ A positional numbering system is a numeral system in which the contribution of a digit to the value of a number is the product of the value of the digit by a factor determined by the position of the digit. In early numeral systems, such as Roman numerals, a digit has only one value: "I" means one, " $\mathrm{X}$ " means ten and "C" a hundred. In modern positional systems, such as the decimal system, the position of the digit means that its value must be multiplied by some value: in " 555 ", the three identical symbols represent five hundreds, five tens, and five units, respectively, due to their different positions in the digit string. 
The offered 'fixing-the-reference' theory is not

sensitive to the difference between non-positional numbering systems and positional Arabic numeral systems. This seems to be a potential problem for the current strategy, which seems to support a realist position since its success depends on a satisfactory reference theory for Arabic numerals. It would be suspicious that a general theory of reference for almost any numbering system can provide a solution to the epistemological problem. (Think of the arithmetic limitations we have when using Roman numerals.)

\subsection{Adjusting the Referentialist's move}

According to Gómez-Torrente, numbers, as the plural cardinality properties they are likely to be, need not stand in any quasi-graphical structural relationship with the numerals (p.13). They may not need to, however, they do stand in a relation like that with Arabic numerals, which plays an important role in this particular way of answering the epistemological question. It is partially in virtue of that relation that we entertain de re thoughts toward numbers via Arabic numerals. The potential move that I outline here is intended to 'complete' a successful Referentialist move. The aim is that the procedure for fixing the reference of the Arabic numerals also captures their morphological property of being positional.

Firstly, the Number rule needs the referents of at least two symbols to make sense of the fact that a symbol's position in a complex numeral is related to its value. The natural way to do so is adding the condition that ' 0 ' refers to the number zero'. The second part of the Number rule could be more difficult to complement, but some observations derived from the fact that 5 As Gómez-Torrente mentions in the chapter, there are many
alternative ways to fix the reference of ' 0 '.

Manuscrito - Rev. Int. Fil. Campinas, v. 43, n. 4, pp. 142-164, Oct.-Dec. 2020. 
numbers are cardinality properties ofpluralities may help (This would further support the view that numbers are plural properties, then we have an intuitive ontology that can underwrite a sensible account of reference fixing for Arabic numerals.):

A plurality $a$ with the property of having $n$ as its number also has the property of admitting partitions as follows,

- For each number $m,(n m>1)$ there is a partition of $a$ such that at least one of its 'sub- pluralities' has $m$ as its number. The symbol placed in the first position from left to right is the numeral that corresponds to the number of pluralities whose cardinality is $m$. If there are $m$ sub-pluralities whose cardinality is $m$, a new position on the right is generated in the corresponding Arabic numeral in base $m$. The symbol placed in the new position is the numeral that corresponds to the number of the pluralities left whose cardinality is 1 , which is less than $m$.

- If there are $m$ times $m$ sub-pluralities whose cardinality is $m$, a new position is generated on the right in the corresponding Arabic numeral in base $m$. The symbol placed in the first position is the numeral corresponding to the number of sub-pluralities whose cardinality is $m$ times $m$. If there are ( $m$ times $m)+k$ sub-pluralities whose cardinality is $m(k<m$ times $m$ ), the symbol placed in the second position is the numeral that corresponds to the number of the pluralities left whose cardinality is $m$, (i.e., ( $m$ times $m+k)-(m$ times $m)=k)$. The symbol placed in the third position is the numeral that corresponds to the number of pluralities whose cardinality is 1 , which is less than $m$.

- If there are $m$ times ( $m$ times $m) \cdots$ 
Certainly, this process has been described by 'brute force'. The ideal would be to present the general rule by which this process occurs in terms of pluralities; I am quite confident that in the right space this task can be accomplished, especially if the rule is centered on a fixed base (preferably base 10). For now, let's use an example to illustrate the broad idea,

Let $a$ be a plurality whose cardinality is $n=5$ and $m$ the number that determines the base of the Arabic numeral system such that $1<m \leq n^{6}$ :

- $\quad$ For $m=n$, the partition in play has 1 plurality whose cardinality is five and 0 pluralities whose cardinalities are less than five. The corresponding Arabic numeral in base five is ' 10 '.

- For $m=4$, the partition in play has 1 plurality whose cardinality is four and 1 remaining plurality whose cardinality is one. The corresponding Arabic numeral in base four is ' 11 '.

- $\quad$ For $m=3$, the partition in play has 1 plurality whose cardinality is three and 2 remaining pluralities whose cardinality is one. The corresponding Arabic numeral in base three is ' 12 '.

- For $m=2$, the partition in play has 1 two-times- two plurality whose cardinality is two, 0 remaining pluralities whose cardinality is greater than two times two, and 1 remaining plurality whose cardinality is one. The corresponding Arabic numeral in base two

\footnotetext{
${ }^{6}$ If $m$ is a base greater than $n=5$, there are no sub-pluralities whose cardinality is $\mathrm{m}$, therefore the corresponding Arabic nu- meral is not complex.
} 
is ' 101 '?

The resulting principle may not be as simple as the Number rule, but this does not imply that it is too conceptually demanding for a competent user of Arabic numerals. All she needs, in addition to the other abilities mentioned, is the ability to associate the idea that a not-too-large plurality can be partitioned in a certain way with the idea that the position of a symbolin a complex Arabic numeral is related to its value. The speakermasters the procedure because of the same reason as in the original account: she develops the ability (related to counting) to systematically associate Arabic numerals, within a given Arabic positional numeral system, to the corresponding numbers.

\section{GAME OVER...?!: CONCLUDING REMARKS}

Gómez-Torrente's theory hypothesizes an association between the Arabic numerals and a system of unsophisticated reference-fixing phrases that single out the corresponding numbers. The ability to generate the series of Arabic numerals underlies this association and is responsible for giving to the speakers the non- descriptive cognitive contact with the referents of the Arabic numerals that would seem required in view of the existence of de re attitudes toward numbers via those numerals.

The reference-fixing theory, along with the epistemic and ontological remarks, does justice to our use of Arabic numerals as well as to a variety of intuitions about numbers. Adding the observation that the property of being part of a positional system of numerals plays a crucial role in the contact we have

${ }^{7}$ Note that a plurality of cardinality 5 contains two sub- pluralities whose cardinality is two. As a result, a new position is generated on the right in the corresponding Arabic numeral in base two.

Manuscrito - Rev. Int. Fil. Campinas, v. 43, n. 4, pp. 142-164, Oct.-Dec. 2020. 
with numbers through Arabic numerals, I maintain that the proposal successfully accounts for the followingintuitions:

1. The cardinality property of a plurality (i.e., its number) is an aspect of it that is common to other pluralities that can be counted by means of the same Arabic numeral

2. The cardinality properties (numbers) are homogeneous in nature and things that are generable from an initial item by recursive application of an homogeneous operation that can plausibly be seen to correspond to the intuitive operation of adding one. It is no coincidence that this is the case with the Arabic numerals system

3. The cardinality property (number) of a plurality can be splitup according to the following fact: the plurality can be partitioned into smaller pluralities, each of which corresponds to an Arabic numeral. The relation between the cardinality properties of the sub-pluralities and the cardinality property of the total plurality is associated with the relation between each of the constituents and the complex Arabic numeral that corresponds to the total plurality. The way these partitions can be selected is reflected in the morphology of the corresponding Arabic numeral. In particular, in the value that each constituent has, according to itsposition.

The epistemological concern of the traditional Antirealist has been addressed from a completely new perspective. As it is explained by Gómez-Torrente: by the time a typical speaker is exposed in earnest to the Arabic numerals, she has learned to recite at least a moderately large initial segment of the sequence of existing verbal numerals and has learned to count pluralities of objects with it. The development of these abilities precedes 
the grasp of the concept of quantity or number as such. The mastery of these abilities eventually induces the appearance of particular conceptions of the numbers in a progressively larger set, and also of a minimal conception of the general notion of number. A system of numerals with suitable properties, such as the Arabic decimal system, plays a crucial role in the evolution towards mastering such abilities.

I have no doubt that the game is not over yet and the Antirealist (or the Descriptivist) will have more to say. Now it is her move.

\section{REFERENCES}

[1] Ackerman, D. "De Re Propositional Attitudes Toward Integers". In: Southwestern Journal of Philosophy, IX, pp. 145-153, 1978.

[2] Balaguer, M. Platonism and Anti-Platonism in Mathematics. Oxford: Oxford University Press, 1998.

[3] Bueno, O. "Modal Epistemology and Mathematical Epistemology". In FISCHER, R. and LEÓN, F. (eds). Modal Epistemology After Rationalism, pp. 67-83. Dordrecht: Springer, 2016.

[4] Burgess, J. and Rosen, G. A Subject With No Object. Oxford: Oxford University Press,1997.

[5] FIELD, H. Realism, Mathematics, and Modality.

Oxford: Blackwell, 1989.

[6] Frege, G.Foundations of Arithmetic. Halle: L. Nebert, 1884.

[7] GómEZ-Torrente, M. "On the Essence and Identity of Numbers". In: Theoria, XXX, pp. 317329, 2015. 
[8] Gómez-Torrente, M. Roads to Reference: An Essay on Reference Fixing in Natural Language. Oxford: Oxford University Press, 2019.

[9] Groenendij, J. and StOKHOF, M. "Questions". In vAN BENTHEM, J. and TER MEulen, A. (eds). Handbook of Logic and Language, pp. 1055-1124. Amsterdam: Elsevier Science, 1997.

[10] Hale, B. and Wright, C. "The Metaontology of Abstraction". In ChALMERS, D. J., MANLEY, D. and WASSERMAN, R. (eds). Metametaphysics, pp. 178-212. Nueva York: Oxford University Press, 2009.

[11] KrifkA, M. "Questions". In MAIEnborn, C. vON Heusinger, K. and PorTNER, P. (eds). Semantics: An International Handbook of Natural Language Meaning, Vol. 2, pp. 1742-1785. Germany: De Gruyter Moton, 2011.

[12] KRIPKE, S. "Logicism, Wittgenstein, and De Re Beliefs About Numbers". Unpublished transcript of the Whitehead Lectures delivered at Harvard University in May of 1992.

[13] Rosen, G. "Nominalism, Naturalism, Epistemic Relativism". In: Philosophical Perspectives, XV, pp. 69-91, 2001.

[14] Wright, C. Frege's Conception of Numbers as Objects. Aberdeen: Aberdeen University Press, 1983.

[15] Yablo, S. "Go Figure: A Path Through Fictionalism". Midwest Studies in Philosophy, XXV, pp. 72 102, 2010.

\section{(cc) BY}

